\title{
Effect of a potential step or impurity on the Bose-Einstein condensate mean field
}

\author{
B. T. Seaman, L. D. Carr, and M. J. Holland \\ JILA, National Institute of Standards and Technology and Department of Physics, \\ University of Colorado, Boulder CO 80309-0440, USA
}

(Dated: October 29, 2018)

\begin{abstract}
The full set of stationary states of the mean field of a Bose-Einstein condensate in the presence of a potential step or point-like impurity are presented in closed analytic form. The nonlinear Schrödinger equation in one dimension is taken as a model. The nonlinear analogs of the continuum of stationary scattering states, as well as evanescent waves, are discussed. The solutions include asymmetric soliton trains and other wavefunctions of novel form, such as a pair of dark solitons bound by an impurity.

PACS numbers: 03.75.Hh, 05.30.Jp, 05.45.Yv
\end{abstract}

\section{INTRODUCTION}

The nonlinear Schrödinger equation (NLS) models many kinds of wave phenomena. The NLS appears in diverse fields such as nonlinear optics 1], gravity waves on deep water 2], magneto-static spin waves [3], solitons in liquid crystals [4], and magneto-sonic solitons in the atmospheric magneto-pause boundary layer [5]. It also describes the dynamics of the mean field of a weakly interacting atomic or molecular Bose-Einstein condensate (BEC) 6, 7, 8, 9], where it is known as the GrossPitaevskii equation 10,11 .

Here, we consider the steady state response of the mean field of a BEC to a potential step or a delta function potential, as modeled by the NLS. These potentials may be easily produced in present experiments on the BEC. The former may be realized by a detuned laser beam shined over a razor edge to make a sharp barrier, where the diffraction-limited fall-off of the laser intensity is smaller than the healing length of the condensate, so that the potential is effectively a step function. The latter models the response of the condensate to an impurity of a length scale smaller than the healing length, which could be realized by a tightly focused laser beam, by another spin state of the same atom, or by any other object, as for instance another alkali atom, confined in an optical trap. Moreover, the solution methods developed in this article may be generalized to arbitrarily complicated piecewise constant potentials.

The complete set of stationary solutions to the NLS with a constant potential on the infinite line were discovered by Zakharov and Shabat 12, 13]. The stationary solutions of the NLS under periodic and box boundary conditions has also been solved analytically [14, 15], as well as the finite well [16]. The parabolic potential has been solved numerically 17]. The potential step has been examined theoretically and experimentally for the linear Schrödinger equation with a constant [18, 19] and oscillating 20 step. Klein examined similar problems experimentally by deflecting neutron beams with a vibrating crystal [21]. In addition, symmetric steady-state solutions with a point-like impurity potential have been studied by Hakim [22] and Taras-Semchuk 23] in cer- tain limiting cases. Point-like impurity potentials have been studied extensively, such as with helium impurities in a BEC 24, 25], BEC formation initiated by point like impurities [26] and impurity scattering in a BEC of sodium 27]. The superfluid transmission of matter waves across various potentials has been studied [28, 29]. In addition, bound solutions to the NLS are the onedimensional analog to the pinned vortex solutions which occur when a discontinuity is present in a two dimensional system, such as in a two dimensional high- $T_{c}$ superconducting system.

In order to obtain the full set of stationary states in closed analytic form, we assume the BEC to be in the quasi-one-dimensional regime. When the transverse dimensions of the BEC are on the order of its healing length, and its longitudinal dimension is much longer than its transverse ones, the 1D limit of the 3D NLS is appropriate to describe the system [30]. The 1D NLS, with an external potential, $V(x)$, may be written

$$
i \partial_{t} \Psi=-\frac{1}{2} \partial_{x x} \Psi+g|\Psi|^{2} \Psi+V(x) \Psi
$$

where a harmonic oscillator confinement in the transverse directions with frequency $\omega$ has been assumed 31 for atoms of mass $\mathrm{M}$, the length has been rescaled according to units of the oscillator length, $l_{h o}=(\hbar / M \omega)^{1 / 2}$, and energy rescaled according to units of the oscillator energy, $\hbar \omega$. The renormalized 1D coupling, $g \equiv 2 a_{s}$, where $a_{s}$ is the $s$-wave scattering length, characterizes the short-ranged pairwise interactions between atoms. The wave function or order parameter $\Psi(x, t)$ has the physical meaning of $\Psi(x, t)=\sqrt{\rho(x, t)} \exp [i \phi(x, t)]$, where $\rho(x, t)$ is the longitudinal line density and the longitudinal superfluid velocity is given by $v(x, t)=\partial \phi(x, t) / \partial x$. Both attractive and repulsive atomic interactions, i.e., $g>0$ and $g<0$, shall be considered.

In the case where the harmonic oscillator length approaches the $s$-wave scattering length, $l_{h o} \simeq a_{s}$, the $1 \mathrm{D}$ NLS no longer models the system and a one-dimensional field theory with the appropriate effective coupling constant must be considered instead [31]. Since $a_{s}$ is on the order of angstroms for typical BEC's, this regime is not relevant to the present study. Thus, it should be noted 
that this study does not examine the Tonks-Girardeau regime [32], where quantum fluctuations become important and the Gross-Pitaevskii equation no longer models the system.

With the experimental demonstration of Feshbach resonances in BEC's of dilute atomic gases [33, 34], it is possible to alter the $s$-wave scattering length and, hence, the nonlinearity of the NLS. Near a Feshbach resonance, the scattering length becomes a function of a uniform background magnetic field. By altering the magnetic field, the effects of the nonlinearity can be experimentally controlled. It is therefore important to be able to characterize the complete set of solutions as a function of the interaction strength.

This article is organized as follows. In Sec. III the full set of stationary solutions of the NLS along the infinite line are presented. In Sec. III the stationary solutions influenced by a step potential are discussed. The effects of a point-like impurity are presented in Sec. IV Section $\nabla$ shows the connection between the NLS solutions and the well-known solutions to the linear Schrödinger equation. The results are discussed in Sec. VI including a physical interpretation of all solution variables. In App. A the special functions used throughout the article are briefly reviewed. Finally, in App. B the fact that the solutions discussed in Sec. III are indeed the full set of stationary solutions to the 1D NLS with a constant potential [14, 15] is demonstrated formally.

\section{CONSTANT POTENTIAL}

A brief review is given of the stationary solutions of Eq. (11) of the form,

$$
\Psi(x, t)=R(x) \exp [i \phi(x)] \exp [-i \mu t],
$$

where $\mu$ is the eigenvalue. There are several excellent references which consider stationary solutions in a constant potential 30, 35, 36, 37, 38]. Assuming a constant external potential $V$, the separation of Eq. (10) into real and imaginary parts gives

$$
\begin{aligned}
R \partial_{x x} \phi+2\left(\partial_{x} R\right)\left(\partial_{x} \phi\right) & =0, \\
\frac{1}{2} \partial_{x x} R+\frac{1}{2}\left(\partial_{x} \phi\right)^{2} R+g R^{3}+V R & =\mu R .
\end{aligned}
$$

Equation (3) can be integrated to give

$$
\partial_{x} \phi=\frac{\alpha}{\rho},
$$

where $\alpha$ is an undetermined constant of integration, and $\rho(x)=R(x)^{2}$ is the single-particle density. Substituting Eq. (5) into Eq. (44) and integrating yields

$$
\frac{\mathrm{d} \rho}{\mathrm{d} x}=2 \sqrt{g \rho^{3}-2(\mu-V) \rho^{2}+C \rho-\alpha^{2}} .
$$

Integrating a second time gives

$$
\int \frac{1}{\sqrt{g \rho^{3}-2(\mu-V) \rho^{2}+C \rho-\alpha^{2}}} \mathrm{~d} \rho=2 x+x_{0},
$$

where $C$ and $x_{0}$ are undetermined constants of integration. It is shown in App. B that the only solutions to this integral equation are given by the Jacobian elliptic functions [39, 40]. In App. @ these special functions are briefly reviewed. The most general form of the solution is then given by

$$
\rho=A \operatorname{sn}^{2}\left(b x+x_{0}, k\right)+B,
$$

where $s n$ is one of the Jacobian elliptic functions, $A$ is a density prefactor, $b$ is a translational scaling, $x_{0}$ is a translational offest, $k$ is the elliptic parameter, and $B$ is a vertical density offset. The period of the density is given by $2 K(k) / b$, where $K(k)$ is the complete elliptic integral of the first kind. Since the density is positive definite, the variables are restricted such that $B \geq 0$ and $B+A \geq 0$. In Sec. VI] the relation between these variables and the mean number density, energy density, and momentum density are calculated and discussed for the nonlinear wave of Eq. (8).

It should be noted that since many of the solutions are unbounded, the norm of the wavefunction remains unconstrained. This is in contrast to bound or localized solutions when the normalization,

$$
\int_{-\infty}^{+\infty}(\rho-\bar{\rho}) \mathrm{d} x=1
$$

may be used [36], where $\bar{\rho}$ is the average density. Alternatively, it is possible to use a nonlinear scaling of the position and density, $x \rightarrow a x$ and $\rho \rightarrow a^{-2} \rho$, to scale the maximum density on one side of the boundary to unity. However, throughout this paper the wavefunction remains unnormalized.

One may then determine the variables $\mu, \alpha$, and $k$, in terms of $A, B, b$, and $g$ by substituting Eq. (8) into Eq. (6) and equating powers of the elliptic $s n$ function to give

$$
\begin{aligned}
\mu & =\frac{1}{2}\left(b^{2}+(A+3 B) g\right)+V, \\
\alpha^{2} & =B(A+B)\left(b^{2}+B g\right), \\
k^{2} & =\frac{A}{b^{2}} g .
\end{aligned}
$$

This leaves the eigenvalue, $\mu$, the constant of integration of the phase, $\alpha$, and the elliptic parameter, $k$, determined up to $A, B, b$, and the interaction strength, $g$. Note that the fact that $\alpha$ enters into the equations only as $\alpha^{2}$ implies that all nontrivial phase solutions, i.e., those for which $\alpha \neq 0$, are doubly degenerate, as $\pm \alpha$ lead to the same value of the eigenvalue, $\mu$, without otherwise changing the form of the density or phase. We shall use the term trivial phase to refer to solutions for which the phase is spatially constant.

In the following two sections these results are applied to piecewise constant potentials. In particular, the potential step and the delta function potential are examined. 


\section{POTENTIAL STEP}

In this section the complete set of solutions to the NLS with an external step potential of height $V_{0}$ beginning at $x=0$,

$$
V(x)=V_{0} \theta(x),
$$

are presented analytically, given the solution parameters on the negative $x$, or left, side of the step. In the following two subsections, the general solution to the NLS with a potential step and some particular examples are discussed.

\section{A. General Solution}

Applying the two boundary conditions of continuity of the wave function and continuity of the derivative of the wave function gives the following five conditions,

$$
\begin{aligned}
\rho\left(0^{+}\right) & =\rho\left(0^{-}\right), \\
\partial_{x} \rho\left(0^{+}\right) & =\partial_{x} \rho\left(0^{-}\right), \\
\phi\left(0^{+}\right) & =\phi\left(0^{-}\right)+2 \pi n, \\
\alpha\left(0^{+}\right) & =\alpha\left(0^{-}\right), \\
\mu\left(0^{+}\right) & =\mu\left(0^{-}\right),
\end{aligned}
$$

where $n$ is an integer. The first two conditions represent the continuity of the density, Eq. (14), and the derivative of the density, Eq. (15). The next two conditions represent the continuity of the phase, Eq. (16), and the derivative of the phase, Eq. (17). Equation (18) demands that the eigenvalue be the same on either side of the boundary. Note that in Eq. (10) the eigenvalue on the right hand side is offset by $V_{0}$ as compared to the left hand side. Since $n$ only enters into the phase and does not effect such quantities as the eigenvalue and density, only the $n=0$ state is considered and, consequently, the phase, $\phi$, is continuous across the boundary. However, it is important to note that all solutions are of denumerably infinite degeneracy, according to Eq. (16). Since $\phi$ is given by

$$
\phi(x)=\int_{0}^{x} \frac{\alpha}{\rho(x)} \mathrm{d} x+\text { const. },
$$

continuity in the phase is easily achieved by setting the constant phase shift equal on either side of the boundary and, therefore, Eq. (16) is satisfied.

In the following derivation, it is assumed that the wavefunction parameters on the left side of the step are known completely. Therefore the density prefactor, $A_{L}$, the vertical density offset, $B_{L}$, the translational scaling, $b_{L}$, and the horizontal offset, $x_{0 L}$ are all known, where the $L$ subscribt refers to variables on the left side; an $R$ subscript will refer to variables on the right side. In addition, the experimental parameters of the interaction strength, $g$, and the potential step height, $V_{0}$, are both known. From the variables on the left and Eq. (8), the density at the boundary, $\rho_{L} \equiv \rho\left(0^{-}\right)$, and its derivative at the boundary, $\partial_{x} \rho_{L} \equiv \partial_{x} \rho\left(0^{-}\right)$, can be determined. The eigenvalue, $\mu$, and the phase constant, $\alpha$, can be determined from Eqs. (10) and (11).

From Eqs. (8) and (14) the square of the Jacobian elliptic $s n$ function can be solved for,

$$
\operatorname{sn}^{2}\left(x_{0 R}, \sqrt{\frac{A_{R} g}{b_{R}^{2}}}\right)=\frac{\rho_{L}-B_{R}}{A_{R}},
$$

and, from Eqs. (10) and (18), the horizontal scaling, $b_{R}$,

$$
b_{R}^{2}=2\left(\mu-V_{0}\right)-\left(A_{R}+3 B_{R}\right) g .
$$

These variables are substituted into Eqs. (17) and (15), using Eq. (11), to give

$$
\begin{aligned}
\alpha_{L}^{2}= & B_{R}\left(A_{R}+B_{R}\right) \\
& \times\left[2\left(\mu-V_{0}\right)-\left(A_{R}+2 B_{R}\right) g\right] \\
\left(\partial_{x} \rho_{L}\right)^{2}= & -4\left(B_{R}-\rho_{L}\right)\left(A_{R}+B_{R}-\rho_{L}\right) \\
& \times\left[2\left(\mu-V_{0}\right)-\left(A_{R}+2 B_{R}+\rho_{L}\right) g\right] .
\end{aligned}
$$

Equations (22) and (23) are quadratic in $A_{R}$ and cubic in $B_{R}$ and can be solved analytically for $A_{R}$ and $B_{R}$ to give six solutions. The remaining variables on the right side can then be found by substituting the values of $A_{R}$ and $B_{R}$ into Eq. (21), to find $b_{R}$, and by taking the inverse Jacobi sn function of Eq. (20) to give

$$
x_{0 R}=\operatorname{sn}^{-1}\left(\frac{\rho_{L}-B_{R}}{A_{R}}, \sqrt{\frac{A_{R} g}{b_{R}^{2}}}\right) .
$$

The full solution is then completely known. It is therefore possible to completely describe the system analytically knowing only the parameters on one side of the step. This not only introduces computational ease in evaluating solutions, it also provides all possible solutions, most of which cannot be determined using purely numerical methods. In the following section, specific examples of a $\mathrm{BEC}$ in the presence of a step potential will be examined.

\section{B. Particular Examples}

The solutions to the potential step problem can be divided into two categories. The eigenvalue, $\mu$, can be large enough that particles are free to move across the boundary and the nonlinear analog to a transmitted wave for the linear Schrödinger equation becomes possible. However, if the eigenvalue is too small, then the wavefunction must decay under the step.

When $\mu$ is larger than the effective potential,

$$
V_{\text {eff }}(x)=V_{0}+g \rho(x),
$$

the wave can be transmitted across the boundary. Note, in the case of an attractive interaction, $g<0$, the eigenvalue can be less than the step height, $V_{0}$, and for a repulsive interaction, $g>0$, the eigenvalue must be strictly 

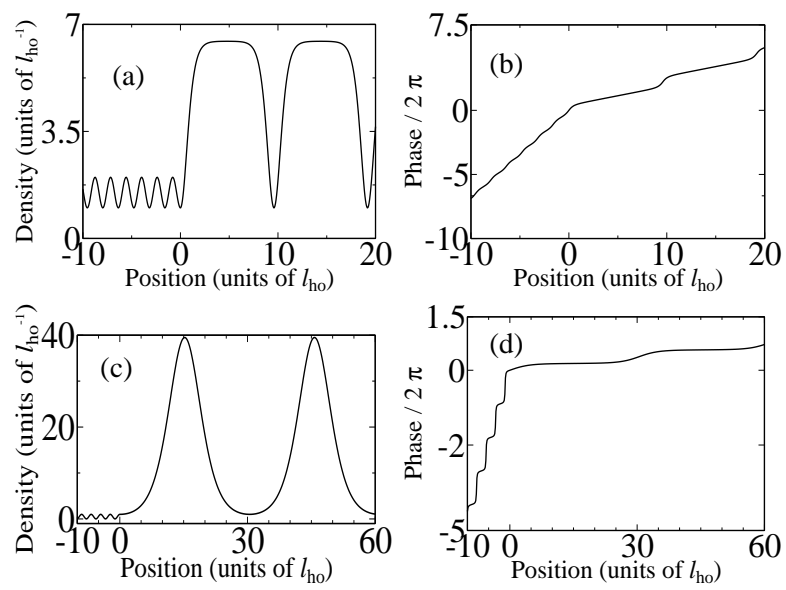

FIG. 1: Stationary solutions to the NLS with a potential step of form $V(x)=V_{0} \theta(x)$. These solutions, which are the nonlinear analogs of the continuum of linear stationary scattering states, exhibit a large deviation from the traditional linear solutions. Shown are particular examples of (a) the density and (b) the phase for a repulsive interaction strength and (c) the density and (d) the phase for attractive interaction strength.

greater than the step height. In Fig. I(a) the density of a nonlinear state with a repulsive interaction strength is shown. A step of height $V_{0}=1$, positioned at $x=0$, and a condensate with an interaction strength of $g=0.2$ and eigenvalue of $\mu=2.404$ were used. Notice that the increased interaction strength and nonlinearity has caused the peaks of the wavefunction to become much broader than in the linear case. The phase that corresponds to this density is shown in Fig. प(b). Figure प(c) shows a similar solution but with an attractive interaction strength. This potential is again given by a step with height of $V_{0}=1$, positioned at $x=0$. An interactionstrength of $g=-0.2$ and eigenvalue of $\mu=0.98$ was used. In this case, the peaks have instead narrowed due to the attractive interaction. The phase that corresponds to this density is shown in Fig. 1(d).

When the eigenvalue is less than the effective potential, $\mu<V_{\text {eff }}(x)$, the wavefunction must decay under the step. In Fig. 2(a) the density of a nonlinear solution with a repulsive interaction strength that decays as it crosses the boundary of the step is shown. A step with height of $V_{0}=1$, positioned at $x=0$, and a condensate with an interaction strength of $g=0.12$ and eigenvalue of $\mu=0.5$ were used. Figure 2(b) shows a similar solution but with an attractive interaction strength. This potential is again given by a step with height of $V_{0}=1$, positioned at $x=0$. An interaction strength of $g=-10$ and eigenvalue of $\mu=-49$ were used. For both wave functions, the phase is necessarily trivial, since all wavefunctions that approach zero at infinity must have $A=-B$, and hence from Eqs. (5) and (11), the phase is constant. In order to stress the importance of the effective potential, and not just the step potential, Figs. 2(c) and (d) show the
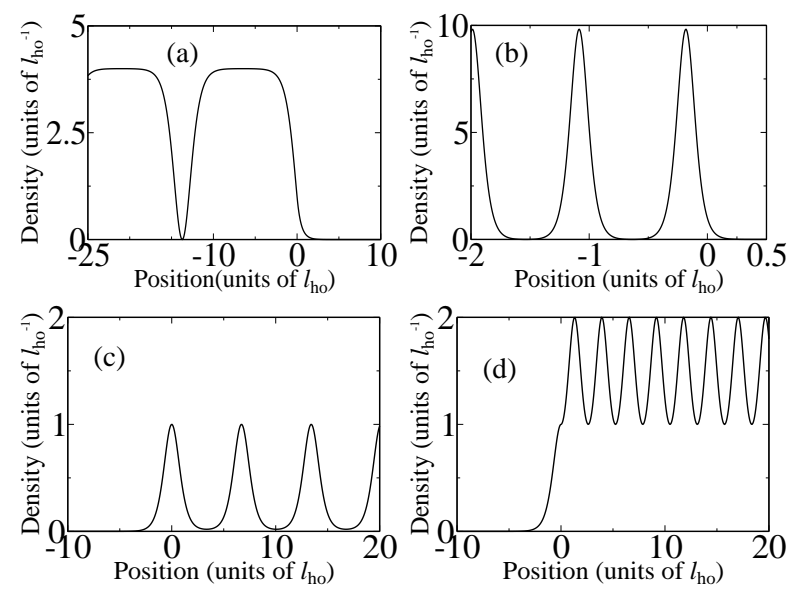

FIG. 2: Stationary solutions to the NLS with a potential step for which the wavefunctions decay, which are the nonlinear analogs to evanescent waves. Shown are particular examples of the densities of nonlinear waves with (a) repulsive interaction strength, (b) attractive interaction strength, and (c) and (d) with an attractive interaction strength that decay on the lower side of the step.

density of two nonlinear wavefunctions with attractive interactions, $g=-1$, that decay on the lower side of the potential. Both wavefunctions have an eigenvalue of $\mu=-0.5$ and a potential of height of $V_{0}=0.01$ and $V_{0}=1$ were used for Fig. 22 (c) and Fig. 22 d), respectively.

Thus the NLS with a potential step has solutions to the step potential that provide a connection between the linear solutions and a wide variety of exotic nonlinear wavefunctions, as shall be discussed in Sec. $\nabla$

\section{POINT-LIKE IMPURITY}

In this section, the case of a potential of form

$$
V(x)=V_{0} \delta(x)
$$

is considered. Such a potential models an impurity which deforms the constant background potential on a length scale much less than that of the healing length. Given the state of the system on the negative $x$, or left side of the impurity, the variables on the right side of the impurity are determined. A negative value of $V_{0}$ represents an attractive impurity, such as due to defects in hydrogen-bonded chains [41, 42], and a positive value of $V_{0}$ represents a repulsive impurity, such as with helium atoms in a BEC [24, 25].

\section{A. General Solution}

The boundary conditions for an impurity are similar to those for the potential step, except that the 
derivative of the wavefunction experiences a discontinuity at the boundary. Therefore, it is necessary that Eqs. (14), (18), (17) and (16) must still be satisfied, as well as

$$
\partial_{x} \rho\left(0^{+}\right)-\partial_{x} \rho\left(0^{-}\right)=-4 \rho(0) V_{0}
$$

It is again assumed that all variables on the left side of the impurity are known as well as the experimental parameters of interaction strength, $g$, and impurity strength, $V_{0}$. Using a treatment that is exactly analogous to that for the step function, all of the parameters on the right side, given those on the left, are analytically determined. The only difference is that in Eqs. (21), (22), (23), and (24), the quantity $\partial_{x} \rho_{L}$ must be replaced with $\left(\partial_{x} \rho_{L}-4 V_{0} \rho_{L}\right)$ and $\left(\mu-V_{0}\right)$ must be replaced with $\mu$. It is therefore possible to completely describe the system analytically knowing only the parameters on one side of the impurity. In the following section, examples of a wavefunction subject to an impurity are examined.

\section{B. Particular Examples}

For the delta function potential, both symmetric and nonsymmetric wavefunctions are possible. Of particular interest are the symmetric wavefunctions in the $k=1$ limit of the Jacobian elliptic functions. In this case all solutions become hyperbolic trigonometric functions with a localized change in the density around the impurity and no oscillations at $\pm \infty$. Solutions of this type we term localized [16]. Due to the form of the solutions, there are four different solution types. The possible wave functions are then

$$
\begin{aligned}
\rho & =\alpha^{2}+\left(1-\alpha^{2}\right) \tanh ^{2}\left(\sqrt{1-\alpha^{2}}|x|+x_{0}\right) \\
\rho & =\alpha^{2}+\left(1-\alpha^{2}\right) \operatorname{coth}^{2}\left(\sqrt{1-\alpha^{2}}|x|+x_{0}\right) \\
\rho & =b^{2} \operatorname{sech}^{2}\left(b|x|+x_{0}\right) \\
\rho & =b^{2} \operatorname{csch}^{2}\left(b|x|+x_{0}\right)
\end{aligned}
$$

where the translational offset, $x_{0}$, is determined by the impurity strength, $V_{0}$, and the density has been normalized according to Eq. (9). Equations (28) and (29) are valid for repulsive interactions, while Eqs. (30) and (31) are valid for attractive interactions.

Figure 3 shows the possible wavefunctions for repulsive interactions. An example of the solution described by Eq. (28) is plotted in Fig. 3(a) with a repulsive impurity of strength $V_{0}=0.5$. This may be interpreted as a single dark soliton bound by an impurity. This is similar to the solution found by Hakim 22] for a soliton that is moving with an impurity. In addition, a bound state of two dark solitons, Fig. 3 (c), can be created when the strength of the impurity is attractive and exactly balances the repulsion between the two dark solitons. Figure 31 (e) shows the hyperbolic cotangent function solution with an impurity strength of $V_{0}=-0.5$; this may be interpreted as a deformation of the ground state constant solution to the NLS with a constant potential. In all plots an interaction strength of $g=1$ and phase constant of $\alpha=0.5$ were used.

It should be noted that there exists a bound state of a repulsive condensate with an attractive impurity. This solution is given by,

$$
\frac{b^{2}}{g} \operatorname{csch}^{2}\left(b x+\operatorname{coth}^{-1}\left(\frac{-V_{0}}{b}\right)\right),
$$

where the interaction strength, $g$ has specifically been included and $b$ must be determined such that the density is normalized to unity. These requirements place a limit on how repulsive the interaction may become and is given by,

$$
g_{\max }=-4 V_{0}
$$

where $g_{\max }$ is the most repulsive interaction the condensate may have. If the interaction is increased past this point, the condensate will spill away from the impurity and will no longer be bound.

The set of symmetric localized solutions for the case of attractive interactions do not allow for nontrivial phases, in contrast to the case of repulsive interactions. For attractive interactions, $g<0$, the hyperbolic secant function solution, Eq. (30), is valid for both $V_{0}>0$, Fig. 4(a), and $V_{0}<0$, Fig. $4(\mathrm{~b})$, where potential strengths of $V_{0}=0.9$ and $V_{0}=-0.9$ were used, respectively. These solutions may be interpreted as a single bright soliton, which is the ground state solution to the 1D-NLS, deformed by an impurity. The hyperbolic cosecant function solution, Eq. (31), is only valid if $V_{0}<0$ and is similar in form to the hyperbolic secant solution of Fig. 4(b). The two solutions types are degenerate for $V_{0}<0$, with an eigenvalue of $\mu=-b^{2} / 2$. In Figs. 世(a) and (b) an interaction strength of $g=-1$ and translational scaling of $b=1$ were used.

Nonsymmetric wavefunctions which oscillate at infinity are also possible and come in two forms: oscillations on one side of the delta function and oscillations on both sides. Figure 5 shows two possible nonsymmetric wavefunctions subject to a delta function positioned at $x=0$ that oscillate on one side of the potential. The density of a wavefunction with a repulsive interaction strength of $g=0.21$ and eigenvalue of $\mu=2.4$, distorted by a delta function, $V_{0}=2$, is shown in Fig. 5(a), where the left side reproduces the hyperbolic tangent function of Eq. (28). In Fig. 5. (b), an attractively interacting, $g=-50$, wavefunction with an eigenvalue of $\mu=-50$, distorted by a delta function, $V_{0}=10$, is shown that appears similar to the evanescent wavefunctions of Fig. 2 that decay beneath a step. Note that Fig. 5(a) has a nontrivial phase while the phase of Fig. 5(b) is trivial.

Figure 6] shows two possible nonsymmetric wavefunctions subject to a delta function positioned at $x=0$ with strength $V_{0}=2$ that oscillate on both sides of the potential. A repulsive interaction strength produces the char- 

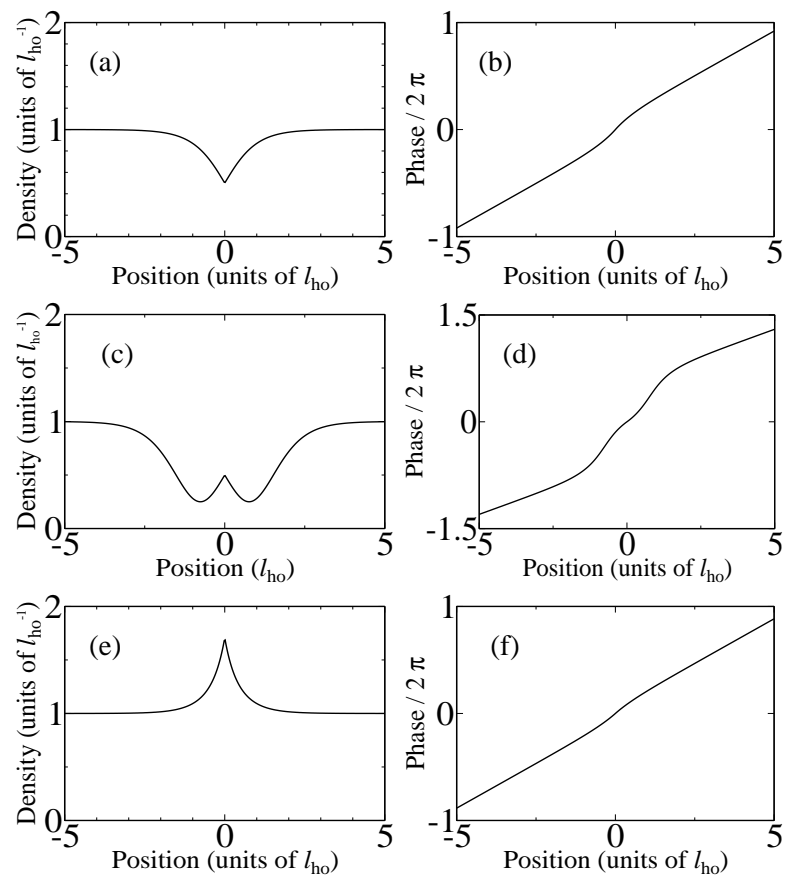

FIG. 3: Localized, symmetric solutions to the NLS with repulsive interaction strength in the presence of an impurity, $V(x)=V_{0} \delta(x)$. Shown are particular examples of (a) the density and (b) the phase of a dark soliton bound by a repulsive impurity, (c) the density and (d) the phase of a pair of dark solitons bound by an attractive impurity, and (e) the density and (f) the phase of a supercurrent deformed by an attractive impurity. Note that (a) and (b) may also be interpreted as deformations of a supercurrent.
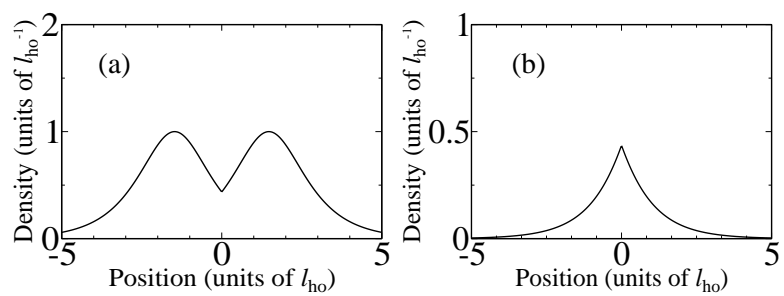

FIG. 4: Localized, symmetric solutions to the NLS with attractive interaction strength in the presence of an impurity. Shown are particular examples of (a) the density of a bright soliton, which is the ground state solution to the NLS, deformed by a repulsive impurity and (b) the density of a bright soliton deformed by an attractive impurity.

acteristic widening of the pulse peaks, Fig. [6(a). The corresponding phase is given in Fig. 6(b). An attractive interaction strength creates a narrowing of the pulse peaks, Fig. [(c). The corresponding phase is given in Fig. [d (d). The condensates in Figs. 6(a) and (c) are characterized by eigenvalues of $\mu=2.4$ and $\mu=-1.3$, respectively.
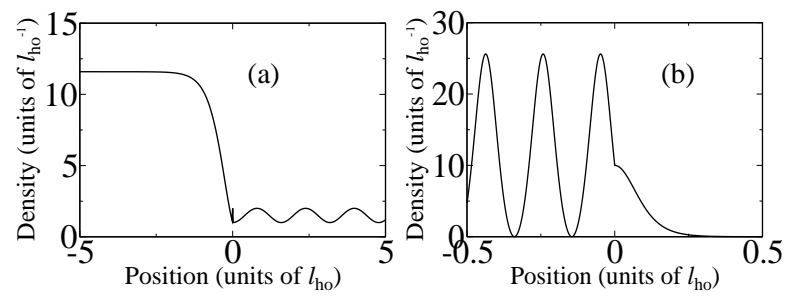

FIG. 5: Solutions to the NLS with an impurity that oscillate on one side of the potential. Shown are particular examples of (a) the density of a nonlinear wave with repulsive interaction strength and (b) the density of a nonlinear wave with attractive interaction strength. These waves have no analog with the solutions of the linear Schrödinger equation.
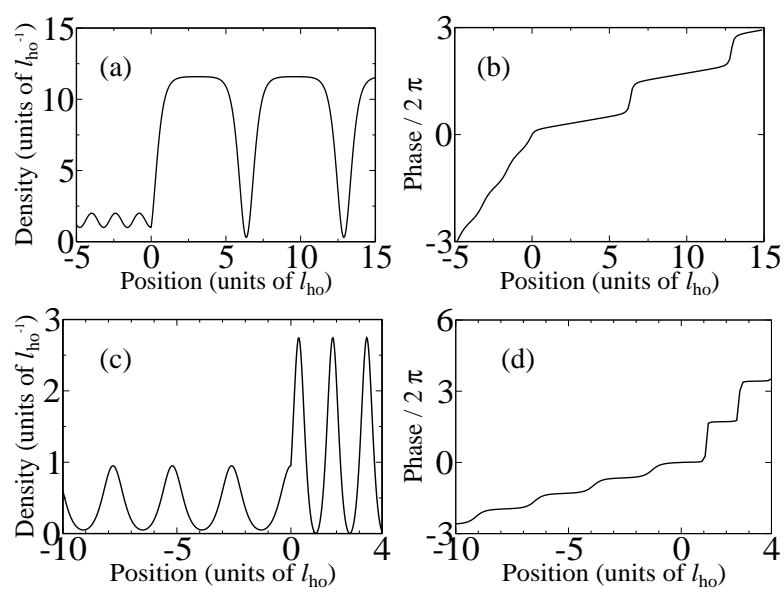

FIG. 6: Nonsymmetric solutions to the NLS with an impurity. Shown are particular examples of (a) the density and (b) the phase of a nonlinear wave with repulsive interaction strength and (c) the density and (d) the phase of a nonlinear wave with attractive interaction strength. These solutions are the nonlinear analogs to the continuum of linear stationary scattering states.

\section{LINEAR LIMITS}

In this section it is shown how the solutions to the NLS connect to the solutions of the linear Schrödinger equation. There are two distinct types of possible waves. If the wave has enough energy it is possible to make the wave propagate through space. However, if it does not have enough energy, the wave can carry no current and is refered to as an evanescent wave. These waves must decay. In the next two sections, these two types of waves are discussed concerning their role as the linear limit of the nonlinear solutions.

\section{A. Transmitted Waves}

In a linear system, if the energy of the system is greater than the potential energy, then the wave can be transmit- 
ted through space as sine waves. The nonlinear Jacobian elliptic $s n$ function waves are the nonlinear analogue of the sine waves of a linear system. In this section, it is shown how the nonlinear solutions, in the linear limit, recreate the linear solutions of propagating waves.

For the linear case, the usual representation of the system by incident, transmitted, and reflected waves is given by,

$$
\begin{aligned}
\Psi_{L}(x, t) & =\left(e^{i k_{L} x}+R e^{-i k_{L} x}\right) e^{-i \mu t}, \\
\Psi_{R}(x, t) & =T e^{i k_{R} x} e^{-i \mu t},
\end{aligned}
$$

where $k_{L}=\sqrt{2\left(\mu-V_{L}\right)}, k_{R}=\sqrt{2\left(\mu-V_{R}\right)}, \mu$ is the eigenvalue of the Schrödinger equation, $R$ is the reflection coefficient, $T$ is the transmission coefficient, and the incident wave is assumed to be coming in from the left. The potentials, $V_{L}$ and $V_{R}$, and the scattering coefficients, $R$ and $T$, are determined by the type of boundary. For the case of a potential step, the potentials are given by $V_{L}=0$ and $V_{R}=V_{0}$. For a delta function potential, the potentials are given by $V_{L}=V_{R}=0$. The wavefunction can be alternately described by an amplitude and phase as follows:

$$
\begin{aligned}
\rho_{L} & =(1+r)^{2}-4 r \sin ^{2}(\sqrt{2 \mu} x-s / 2), \\
\partial_{x} \phi_{L} & =\frac{(r-1)(r+1) \sqrt{2 \mu}}{(1+r)^{2}-4 r \sin ^{2}(\sqrt{2 \mu} x-s / 2)},
\end{aligned}
$$

where

$$
\begin{aligned}
\Psi_{L}(x, t) & =\sqrt{\rho_{L}(x)} \exp \left[i \phi_{L}(x)\right] e^{-i \mu t} \\
R & =r e^{i s}
\end{aligned}
$$

with $r$ and $s$ real. The nonlinear solution is then connected to the linear solution by

$$
\begin{aligned}
x_{0} & =-s / 2, \\
b^{2} & =2 \mu, \\
A & =-4 r, \\
B & =(r+1)^{2} .
\end{aligned}
$$

The transmitted wave can easily be given by

$$
\begin{aligned}
\rho_{R} & =t^{2}, \\
\partial_{x} \phi_{R} & =\frac{\sqrt{2 \mu}}{t^{2}},
\end{aligned}
$$

where

$$
\begin{aligned}
\Psi_{R}(x, t) & =\sqrt{\rho_{R}(x)} \exp \left[i \phi_{R}(x)\right] e^{-i \mu t}, \\
T & =t e^{i w},
\end{aligned}
$$

with $t$ and $w$ real. The nonlinear solution is then connected to the linear solution by

$$
\begin{aligned}
x_{0} & =0, \\
b^{2} & =2 \mu, \\
A & =0, \\
B & =t^{2} .
\end{aligned}
$$

The nonlinear solutions when the eigenvalue is greater than the effective potential are therefore adiabatically connected to the linear transmitted wave solutions. The next section connects the decaying evanescent waves with nonlinear solutions.

\section{B. Evanescent Waves}

If the eigenvalue, $\mu$, is less than the effective potential then the wave function must decay. In the linear case, the decay is precisely exponential. The Jacobian elliptic sn function solution, Eq. (8), of the NLS can provide the appropriate exponential decay. In the limits $g \rightarrow 0$ and $B, x_{0} \rightarrow+\infty$ under the constraints $A=-B$ and $A g / b^{2}=$ 1, Eq. (8) gives

$$
\lim _{B, x_{0} \rightarrow+\infty} B \operatorname{sech}^{2}\left(b x+x_{0}\right)=\rho_{0} e^{-b x},
$$

where $\rho_{0}$ is the density at the boundary and is given by,

$$
\rho_{0}=\frac{4 B}{e^{2 x_{0}}} .
$$

When the limit that $g$ approach zero is not enforced,

$$
\rho(x) \propto \frac{1}{\left(N_{+} \exp ^{+b x}+N_{-} \exp ^{-b x}\right)^{2}},
$$

where $N_{+}$and $N_{-}$are constants related to the magnitude and sign of the interaction strength. The decay of the density is, therefore, not strictly an exponential decay. It is interesting to note that all decaying solutions, whether linear or nonlinear, must possess a trivial phase. This is due to the restriction that $A=-B$, and, hence from Eqs. (11) and (5), the phase must vanish. This is also consistent with the physical interpretation of the phase since a nontrivial phase corresponds to a superfluid velocity and the velocity must vanish if the wave cannot be transmitted.

\section{DISCUSSION AND CONCLUSIONS}

The full set of stationary states of the mean field of a Bose-Einstein condensate, modeled by the nonlinear Schrödinger equation in one dimension, in the presence of a potential step or pointlike impurity were presented in closed analytic form. Non-decaying solutions were divided into two categories: localized soliton-like solutions, and solutions that oscillate out to infinity. The localized solutions are of a purely nonlinear character, as they have no linear analog. The oscillating solutions, on the other hand, were shown to be adiabatically connected to the solutions to the linear Schrödinger equation.

The localized solutions present novel wavefunctions. With a delta function potential, the localized solution can be interpreted as a single bright or dark soliton trapped 
by the impurity. In addition, it was shown that an impurity can also bind a soliton pair. If the impurity is attractive, the natural repulsion between two dark solitons can be exactly canceled by the attraction of the impurity, while if the impurity is repulsive, it can balance the natural attraction of in-phase bright solitons. Since these solutions are conjected to be stable (see below), they are excellent candidates for the experimental realization of stationary excited states of a Bose-Einstein condensate. In addition, the maximum repulsive interaction strength of the condensate with an attractive impurity that allows for a bound state has been determined.

The oscillating solutions to the NLS, despite being adiabatically connected to oscillating solutions to the Schrödinger equation, have very different properties due to the concept of an effective potential. For the attractive interaction solution for an evanescent wave decaying under a step, as illustrated in Fig. 2(b), the eigenvalue is larger than the effective potential in the regions of high density and is less than the effective potential in regions of low density. Figures 2 (c) and (d) show more radical deviations from the linear solutions since the wavefunctions decay on the lower side of the step.

It is possible to characterize the general solution to the NLS, Eq. [8], in terms of physical quantities such as the mean linear number density, $\bar{n}$, mean energy density, $\overline{\mathcal{E}}$, and mean momentum density, $\overline{\mathcal{P}}$. The densities are given by

$$
\begin{aligned}
& \bar{n}=B+A\left(\frac{1}{k^{2}}-\frac{E(k)}{k^{2} K(k)}\right), \\
& \overline{\mathcal{E}}=\bar{n} \mu+\frac{3 B^{2} g-b^{2} A}{6}+\frac{2}{3}(\mu-V)(\bar{n}-B), \\
& \overline{\mathcal{P}}=\alpha,
\end{aligned}
$$

where $\mu, \alpha$, and $k$ are given by Eqs. (10), (11), and (12), respectively. In general, the number, energy, and momentum densities must be calculated separately for the left and right sides of a boundary. These densities can be used to determine the variables $A, B$, and $b$ of Eq. (8), leaving only the translational offset, $x_{0}$, as a free variable determined by the boundary conditions. It should be noted that the factor multiplying $A$ in the mean number density, Eq. [55), approaches one half when $k$ approaches zero and approaches one when $k$ approaches unity. This is to be expected since the mean number density of a linear wave is given by $B+A / 2$ and the mean number density of an extremely nonlinear wave is given by $B+A$. The mean energy density can easily be calculated from

$$
\overline{\mathcal{E}}=\bar{n} \mu-\frac{g}{2} \overline{\rho^{2}},
$$

and so the second and third terms on the right side of Eq. (56) are due to this nonlinear correction. Since the mean momentum density, Eq. (57), is equal to $\alpha$, the mean momentum density must be equal on both sides of the boundary due to the boundary condition on $\alpha$, Eq. (17). In addition, the momentum density as a func- tion of position is also given by $\alpha$ and so the momentum density is equal everywhere.

The healing length, $\xi$, of the NLS in the quasi-onedimensional regime, where the transverse dimensions are trapped by a harmonic potential of frequency $\omega$, is given by

$$
\xi^{2}=\frac{l_{h o}^{2}}{8 \pi a_{s} \bar{n}}
$$

where $\bar{n}$ is given by Eq. 55.5. Since the mean number density can vary across the boundary, it is possible for the condensate to have a different healing length on either side of a boundary. Since the speed of sound in the condensate is inversely proportional to the healing length, the speed with which phonon-like excitations can travel vary as they cross the boundary.

While finding the complete set of solutions to Eq. (10) with an impurity or step potential provides much information about the system, only stable solutions are experimentally observable. Previous works have examined the stability of stationary states for a constant external potential (see, for example, 35, 36, 37, 38, 43, 44, 45, 46]), as well as for periodic and harmonic potentials (see, for example, [47, 48]). Most studies are ultimately numerical: linear stability can be solved in a few special cases, while nonlinear stability is analytically intractable. For a constant external potential, single bright and dark solitons and dark soliton trains are stable. A finite number of bright solitons may form bound states, as for example order $n$ solitons $(n>1)$. Bright soliton trains are always unstable, but may be experimentally stable, in that their lifetime is much longer than experiments, which typically require stability timescales of from milliseconds to seconds. Bright soliton trains which have a phase difference $\Delta \phi$ between adjacent peaks such that $-\pi / 2<\Delta \phi<3 \pi / 2$ exhibit this experimental stability, with the lifetime being longer the closer $\Delta \phi$ is to $\pi$. Bright soliton trains with $-\pi / 2<\Delta \phi<\pi / 2$ are unstable but become quasi-periodic in time in a finite system.

Based on these known results from the case of a constant potential, as well as the from the stability analysis with an impurity performed by Bogdan et al. 49], the stability of an attractive condensate with an impurity is as follows. According to Bogdan, the bound state of two bright solitons, as in Fig. पl(b), are stable, since, so long as they are strongly overlapping, they will be in phase $(\Delta \phi=0)$ and remain bound to the impurity. This is also the ground state of the system. However, the kind of solution shown in Fig. [4a) is unstable [49]. We then conject on the stability of a repulsive condensate that is not bound and whose density approaches a nonzero constant at infinity. Localized solutions in the case of repulsive nonlinearity are obviously stable in the cases of Fig. 3(a)-(b) and 3(e)-(f), since they are the ground state. The bound pair of dark solitons, illustrated in Fig. 31(c)(d), should be likewise stable, so long as the impurity is sufficiently strong. All of these solution types, except for attractive solitons bound by a repulsive imputiry, are ex- 
pected to be experimentally observable in finite systems, such as an elongated harmonic trap. An excellent analysis of the stability of solitons pinned with impurities is given by Bogdan, et. al. [49].

The stability of soliton trains is a less certain issue. In the repulsive case, the central question is whether or not the phase locking of the individual solitons in the train is destroyed by the impurity or potential step; if it is, they may become unstable in the region of the discontinuity in the potential. In the case of bright soliton trains, unless there is a strong phase difference between the peaks, they will attract and become unstable; otherwise, the discontinuity should not present a source of instability, since bright solitons adjust themselves to perturbation by emission of a small fraction of the total wavefunction [45]. In order to perform numerical studies, the solutions would have to be quantized on a ring, in order to provide a finite domain for simulation. Such a stability study presents a subject for future research.

We emphasize that neither the idea of left and right traveling waves nor that of reflected plus incident waves apply to nonlinear wave equations. This is important since one cannot create wavepackets from linear combinations of these solutions. Instead, these solutions already contain the wavepacket-like solutions, or solitons, that are necessary to describe the system; moreover, solitons, unlike wavepackets, are nondispersive. Time dependent nonlinear scattering remains an open question that can certainly be addressed via numerical studies. In general, the stationary solutions to the NLS give physical insight into its dynamics, without which numerical solutions may be difficult to interpret. Perhaps more importantly, using the general nature of the solutions to the cases of a step function and an impurity, it is possible to describe all stationary states to piecewise constant potentials.

In conclusion, we have analytically solved for all stationary solutions to the nonlinear Schrödinger equation with a delta function or a step function potential. This models the steady state behavior of the mean field of a Bose-Einstein condensate in the presence of an impurity, or of a potential step created by, for instance, a laser passing over the edge of a razor blade. Novel wavefunctions were found, including solitons trapped by the impurity and the nonlinear analog of transmitted and evanescent waves.

\section{Acknowledgments}

We acknowledge helpful discussions with J. Cooper. Support is acknowledged for B.T.S. from the National Science Foundation and for L.D.C. from the U.S. Department of Energy, Office of Basic Energy Sciences via the Chemical Sciences, Geosciences and Biosciences Division, as well as the National Science Foundation via grant no. mps-drf 0104447
TABLE I: Limits of the Jacobian elliptical functions and integrals [0].

\begin{tabular}{|c|c|c|}
\hline & $k=0$ & $k=1$ \\
\hline $\operatorname{sn}(u, k)$ & $\sin (u)$ & $\tanh (u)$ \\
\hline $\operatorname{cn}(u, k)$ & $\cos (u)$ & $\operatorname{sech}(u)$ \\
\hline $\operatorname{dn}(u, k)$ & 1 & $\operatorname{sech}(u)$ \\
\hline $\operatorname{ns}(u, k)$ & $\csc (u)$ & $\operatorname{coth}(u)$ \\
\hline $\operatorname{ds}(u, k)$ & $\csc (u)$ & $\operatorname{csch}(u)$ \\
\hline $\operatorname{cs}(u, k)$ & $\cot (u)$ & $\operatorname{csch}(u)$ \\
\hline $\mathrm{K}(k)$ & $\pi / 2$ & $\infty$ \\
\hline $\mathrm{E}(k)$ & $\pi / 2$ & 1 \\
\hline
\end{tabular}

\section{APPENDIX A: JACOBIAN ELLIPTIC FUNCTIONS}

A brief review of the Jacobian elliptic functions 40, 50. is given. Of the 12 elliptic functions, there are only six that are normalizable. Of these six, only three represent a different physical form, $s n, c n$ and $d n$. However, they are still related by,

$$
\begin{aligned}
\mathrm{cn}^{2} & =1-\mathrm{sn}^{2} \\
\mathrm{dn}^{2} & =1-k^{2} \mathrm{sn}^{2}
\end{aligned}
$$

The six non-normalizable elliptic functions can also be reduced through a phase shift to three with different forms, $n s, d s$, and $c s$, which are also related by,

$$
\begin{aligned}
\mathrm{cs}^{2} & =\mathrm{ns}^{2}-1, \\
\mathrm{ds}^{2} & =\mathrm{ns}^{2}-k^{2} .
\end{aligned}
$$

The normalizable and nonnormalizable functions can be related through

$$
\operatorname{sn}^{2}\left(i K\left(1-k^{2}\right)+z, k\right)=k^{2} \mathrm{~ns}^{2}(z, k),
$$

where $K(x)$ is the complete elliptic integral of the first kind. Therefore the square of any elliptic function can be related linearly to $s n^{2}$.

The limits of the $s n, c n$ and $d n$ functions, along with the complete elliptic integrals $K(k)$ and $E(k)$ are presented in Table The period of the $s n^{2}, c n^{2}$ and $d n^{2}$ functions is $2 K(k)$.

\section{APPENDIX B: COMPLETE SOLUTION SET}

It is possible to prove that Eq. (7) is a Jacobian elliptic integral of the first kind and can therefore be inverted to produce the Jacobian elliptic functions. If arbitrary parameters are used, Eq. (7) becomes,

$$
\int \frac{1}{\sqrt{A_{3} \rho^{3}+A_{2} \rho^{2}+A_{1} \rho+A_{0}}} \mathrm{~d} \rho=x+x_{0},
$$


where the $A_{i}$ 's are real constants. The cubic polynomial can be factored to give,

$$
\int \frac{1}{\sqrt{\left(\rho+B_{1}\right)\left(\rho+B_{2}\right)\left(\rho+B_{3}\right)}} \mathrm{d} \rho=x+x_{0},
$$

where at least one of the constants $B_{i}$ must be real. Without loss of generality we may take the real constant as $B_{1}$. The substitution $\rho=y^{2}-B_{1}$ is then made in Eq. (B2) to yield

$$
\int \frac{2}{\sqrt{\left(y^{2}+\left(B_{2}-B_{1}\right)\right)\left(y^{2}+\left(B_{3}-B_{1}\right)\right)}} \mathrm{d} y=x+x_{0} \text {. }
$$

This is the general form of the elliptic integral of the first kind [40] and therefore gives

$$
C_{1} \mathrm{el}^{-1}\left(C_{2} y, C_{3}\right)=x+x_{0}
$$

where the $C_{i}$ 's are constants and $e l$ is one of the twelve elliptic functions. This can be inverted and $\rho$ replaced to produce

$$
\sqrt{\rho+B_{1}}=C_{2}^{-1} \operatorname{el}\left(C_{1}^{-1}\left(x+x_{0}\right), C_{3}\right),
$$

or finally,

$$
\rho=C_{2}^{-2} \mathrm{el}^{2}\left(C_{1}^{-1}\left(x+x_{0}\right), C_{3}\right)-B_{1} .
$$

Since the square of any elliptic function can be related linearly to the square of $s n$, only one independent solution of the form

$$
\rho=A \operatorname{sn}^{2}\left(b x+x_{0}, k\right)+B
$$

need be considered.
[1] V. I. Talanov, Zh. Eksp. Teor. Fiz. 2, 31 (1965).

[2] H. Hasimoto and H. Ono, J. Phys. Sco. Jpn 33, 805 (1972).

[3] M. M. Scott, Y. K. Fetisov, V. T. Synogach, and C. E. Patton, J. Appl. Phys. 88, 4232 (2000).

[4] C. Conti, M. Peccianti, and G. Assanto, Phys. Rev. Let. 91, 073901 (2003).

[5] K. Stasiewicz, P. K. Shukla, G. Gustafsson, S. Buchert, B. Lavraud, B. Thide, and Z. Klos, Phys. Rev. Let. 90, 085002 (2003).

[6] M. H. Anderson, J. R. Ensher, M. R. Matthews, C. E. Wieman, and E. A. Cornell, Science 269, 198 (1995).

[7] K. B. Davis, M.-O. Mewes, M. R. Andrews, N. J. van Druten, D. S. Durfee, D. M. Kurn, and W. Ketterle, Phys. Rev. Lett. 75, 3969 (1995).

[8] C. C. Bradley, C. A. Sackett, J. J. Tollett, and R. G. Hulet, Phys. Rev. Lett. 75, 1687 (1995).

[9] C. C. Bradley, C. A. Sackett, and R. G. Hulet, Phys. Rev. A 55, 3951 (1997).

[10] E. P. Gross, Nuovo Cimento 20, 454 (1961).

[11] L. P. Pitaevskii, Zh. Eksp. Teor. Fiz. 40, 646 (1961).

[12] V. E. Zakharov and A. B. Shabat, Zh. Eksp. Teor. Fiz. 61, 118 (1971).

[13] V. E. Zakharov and A. B. Shabat, Zh. Eksp. Teor. Fiz. 64, 1627 (1973).

[14] L. D. Carr, C. W. Clark, and W. P. Reinhardt, Phys. Rev. A 62, 063610 (2000).

[15] L. D. Carr, C. W. Clark, and W. P. Reinhardt, Phys. Rev. A 62, 063611 (2000).

[16] L. D. Carr, K. W. Mahmud, and W. P. Reinhardt, Phys. Rev. A 64, 033603 (2001).

[17] Y. S. Kivshar, T. J. Alexander, and S. K. Turitsyn, Phys. Lett. A 278, 225 (2001).

[18] J. Villavicencio, R. Romo, and S. S. y. Silva, Phys. Rev. A 66, 042110 (2002).

[19] J. G. Muga and M. Buttiker, Phys. Rev. A 62, 023808 (2000).

[20] J. Felber, R. Gahler, and C. Rausch, Phys. Rev. A 53, 319 (1996).

[21] A. G. Klein, P. Prager, P. J. Wagenfeld, H.and Ellis, and
T. M. Sabine, Appl. Phys. Lett. 10, 294 (1967).

[22] V. Hakim, Phys. Rev. E 55, 2835 (1997).

[23] D. Taras-Semchuk and J. M. F. Gunn, Phys. Rev. B 60, 13139 (1999).

[24] P. Capuzzi and E. S. Hernandez, Phys. Rev. S 62, 023603 (2000).

[25] J. Harms and J. P. Toennies, Phys. Rev. Let. 83, 344 (1998).

[26] R. M. Cavalcanti, P. Giacconi, G. Pupillo, and R. Soldati, Phys. Rev. A 65, 053606 (2002).

[27] A. P. Chikkatur, A. Gorlitz, D. M. Stamper-Kurn, S. Inouye, S. Gupta, and W. Ketterle, Phys. Rev. Let. 85, $483(2000)$.

[28] P. Leboeuf, N. Pavloff, and S. Sinha, Phys. Rev. A 68, 063608 (2003).

[29] N. Pavloff, Phys. Rev. A 66, 013610 (2002).

[30] L. D. Carr, M. A. Leung, and W. P. Reinhardt, J. Phys. B 33, 3983 (2000).

[31] M. Olshanii, Phys. Rev. Lett. 81, 938 (1998).

[32] M. Girardeau, J. Math. Phys. 1, 516 (1960).

[33] J. L. Roberts, N. R. Claussen, J. P. Burke, Jr., C. H. Greene, E. A. Cornell, and C. E. Wieman, Phys. Rev. Let. 81, 5109 (1998).

[34] A. Inouye, M. R. Andrews, J. Stenger, H.-J. Miesner, D. M. Stamper-Kurn, and W. Ketterle, Nature 392, 151 (1998).

[35] E. Infeld and G. Rowlands, Nonlinear Waves, Solitons and Chaos, 2nd ed. (Cambridge University Press, United Kingdom, 2000).

[36] Y. S. Kivshar and B. Luther-Davies, Physics Reports 298, 81 (1998).

[37] E. Infeld and G. Rowlands, J. Phys. A 12, 2255 (1979).

[38] H. C. Yuen and M. Lake, Bruce, Phys. Fluids 18, 956 (1975).

[39] F. Bowman, Introduction to Elliptic Functions, with Applications (Dover, New York, 1961).

[40] Handbook of Mathematical Functions, edited by M. Abramowitz and I. A. Stegun (National Bureau of Standards, Washington, D.C., 1964).

[41] Y. S. Kivshar, Z. Fei, and L. Luis Vzquez, Phys. Rev. 
Lett. 67, 1177 (1991).

[42] Y. S. Kivshar, Phys. Rev. A 43, 3117 (1991).

[43] J. P. Gordon, Opt. Lett. 8, 596 (1983).

[44] J. P. Gordon and H. A. Haus, Opt. Lett. 11, 665 (1986).

[45] H. Haus and W. S. Wong, Rev. Mod. Phys. 68, 423 (1996).

[46] C. Sulem and P. L. Sulem, Nonlinear Schrödinger Equations: Self-focusing Instability and Wave Collapse (Springer-Verlag, New York, 1999).
[47] J. C. Bronski, L. D. Carr, B. Deconinck, J. N. Kutz, and K. Promislow, Phys. Rev. E 63, 036612 (2001).

[48] L. D. Carr, J. N. Kutz, and W. P. Reinhardt, Phys. Rev. E 63, 066604 (2001)

[49] M. M. Bogdan, A. S. Kovalev, and I. V. Gerasimchuk, Low Temp. Phys. 23, 145 (1997).

[50] L. M. Milne-Thomson, Jacobian Elliptic Function Tables (Dover Publications Inc., New York, 1950). 